\title{
PERSPECTIVES
}

OPINION

\section{Can landscape ecology untangle the complexity of antibiotic resistance?}

Randall S. Singer, Michael P. Ward and George Maldonado

Abstract | Bacterial resistance to antibiotics continues to pose a serious threat to human and animal health. Given the considerable spatial and temporal heterogeneity in the distribution of resistance and the factors that affect its evolution, dissemination and persistence, we argue that antibiotic resistance must be viewed as an ecological problem. A fundamental difficulty in assessing the causal relationship between antibiotic use and resistance is the confounding influence of geography: the co-localization of resistant bacterial species with antibiotic use does not necessarily imply causation and could represent the presence of environmental conditions and factors that have independently contributed to the occurrence of resistance. Here, we show how landscape ecology, which links the biotic and abiotic factors of an ecosystem, might help to untangle the complexity of antibiotic resistance and improve the interpretation of ecological studies.

Bacterial resistance to antibiotics continues to pose a serious threat to human and animal health. The relationship between antibiotic use and the development of resistance has been studied extensively, with some of this research aimed at identifying antibiotic treatment strategies that minimize the maintenance of resistance genes and resistant bacterial strains. However, attempts to reduce resistance levels through changes in antibiotic use patterns have had variable success, leading us to question whether antibiotic use is the only (or even the predominant) selection pressure maintaining antibiotic resistance $^{1-9}$.

We often assume that antibiotics are human-made compounds that have the unfortunate consequence of promoting antibiotic resistance. In reality, many antibiotics, including many with clinical relevance, are naturally produced by microorganisms in the environment ${ }^{10}$. The function of these naturally produced small molecules is still not entirely understood ${ }^{11,12}$, but might include roles such as bacterial communication through cell-to-cell signalling ${ }^{12-14}$. These naturally produced compounds can have an impact on microbial populations in many ways, including selection for populations that possess mechanisms of resistance to these small molecules ${ }^{12,13}$. The presence of naturally produced antibiotics in the environment is rarely considered as a potential contributor to the resistance observed in studies of antibiotic resistance. Antibiotic-resistance genes and antibioticresistant microorganisms can be found in areas with little to no obvious anthropogenic influence, and in samples that predate the use of human-produced antibiotics. Clearly there is a background level of resistance that must be accounted for in our studies of resistance ${ }^{15}$.

In addition to exposure to naturally produced antibiotics, bacteria at a given site can be exposed to antibiotics that have little relation to the antibiotics that are actually used at that location. Humanproduced antibiotics and their metabolites can be found in the effluents of hospitals and pharmaceutical plants, and in agricultural runoff ${ }^{15-17}$. Consequently, antibiotics can be found in groundwater, surface water and in the soil ${ }^{18}$. Studies that attempt to relate the use of antibiotics in human and veterinary medicine to resistance levels but that ignore the potential for environmental selection might be confounded.

The historical dogma was that antibiotic resistance should not be found in areas where antibiotics have not been used. The presence of resistance was thought to exert a fitness cost on the organism: when the antibiotic was removed, the organism was expected to lose its resistance or else be competitively replaced by a susceptible organism with higher fitness. This assumption has been questioned ${ }^{19-21}$, and recent evidence demonstrates that, in the absence of selection pressure due to antibiotic use, resistance levels in bacterial populations are often slow to decline, if at all ${ }^{5,7,22}$. In fact, some organisms with antibiotic-resistant phenotypes and genotypes seem to be fitter than their susceptible counterparts ${ }^{23-26}$, and compensatory mutations have been observed that allow the resistant organism to retain its fitness level ${ }^{27}$. Many potential selection forces for resistance have been described, including antibiotics, metals and other compounds such as disinfectants. These selection forces might function through a diversity of genetic linkages in the bacterial cell ${ }^{3}$. Therefore, predictions of changes in antibiotic-resistance levels require considerable information about bacterial genetics and the diversity of co-selection processes that exist. Antibiotic resistance can also be spread through the transmission and dissemination of antibiotic-resistant organisms, potentially over large geographical distances. To assess the causes of resistance and to design strategies to reduce the impacts of resistance, we must include measurements of the many nonantibiotic causes of resistance and begin to understand the relative importance of the background pool of resistance.

Landscape ecology focuses on the influence of spatial heterogeneity on ecological processes and, specifically, addresses the distribution of biotic and abiotic factors in an ecosystem to evaluate the causes and consequences of these distributions ${ }^{28}$. It is ideally suited to assessing the many 
environmental factors that can influence antibiotic resistance. Landscape ecology is related to biogeography, which deals with the factors in an ecosystem that affect the diversity and abundance of living organisms. A recent comprehensive review of microbial biogeography argues convincingly that microbial population structure in the environment is non-random, implying that there are environmental processes that are partly responsible for this spatial variation in microbial diversity ${ }^{29}$. Because of this variation, the spatial scale at which the microbial data are collected, and the spatial selection of study groups, can influence the conclusions of a study ${ }^{29}$.

With respect to microorganisms and disease, the influence of the environment on the transmission of infectious diseases has been appreciated for some time ${ }^{30}$. Relevant data that could be included in an analysis of the environment and infectious disease might include the location of sampling, the distribution of hosts that are capable of harbouring the microorganisms in question, the spatial determinants of the transmission or selection of these microorganisms (for example, air, water, dissemination by wildlife), and any constraints to movement or dissemination of the microorganisms. These approaches have been incorporated into the study of various vector-borne diseases to predict risks in relation to specific geographical factors $^{31-33}$. Unfortunately, the approaches of landscape ecology have rarely been applied to studies of antibiotic resistance, and then only in a superficial manner. For example, a recent study found higher levels of resistant bacteria and resistance genes in anthropogenically impacted sites versus pristine sites ${ }^{34}$. This study did not attempt to determine the causal factors for this difference between the sites. When resistance levels and antibiotic uses are low in one area but higher in other areas it is easy to conclude that the low usage levels have resulted in the low observed resistance levels ${ }^{35}$. However, this appealing conclusion is not necessarily the most accurate.

A different approach to relating environmental variables to antibiotic resistance can be seen in a study of the spatial variation of antibiotic interactions and resistance among streptomycetes in a $1 \mathrm{~m}^{2}$ plot of prairie soil ${ }^{36}$. This study found that the distribution of antibiotic-inhibitory activity was non-uniform, indicating the potential for extreme spatial heterogeneity in the selection pressures in soils. Resistance among the streptomycetes was more uniform, suggesting that resistance provides more benefits than inhibition or that the fitness costs of maintaining resistance are negligible in the absence of selection. If this approach to examining alternative causes of resistance was to be applied to larger ecological investigations of resistance we would need to collect additional data about the distributions of the various selection pressures and routes of transmission over spatial and temporal scales. These data can then be combined with antibiotic-resistance data to help understand the ecological dynamics of resistance and facilitate the prediction of strategies to minimize the impact of resistance.

\section{...the approaches of landscape ecology have rarely been applied to studies of antibiotic resistance...}

When the focus of a study is to relate the impact of the use of an antibiotic to resistance to that antibiotic, incorporating the tools of landscape ecology will aid in both improving study design and analyzing study data more accurately. Studies that address the complex array of selection pressures, routes of transmission and background levels of resistance that exist in an ecosystem can be designed to improve our understanding of the dynamics of resistance. The goal of this paper is to demonstrate how landscape ecology can be incorporated into studies of antibiotic resistance to provide conclusions that are more accurate, more informative and closer to the actual causal relationships. We will describe the challenges inherent in quantifying the causal relationship between antibiotic use and resistance, particularly for those studies that assess resistance patterns and the factors that can influence these patterns over broad spatial and temporal scales, and in complex environments. We will propose an approach to account for the ecological complexity of antibiotic resistance and we will show how it is relevant to both study design and data analysis.

\section{Controls and confounding bias}

When antibiotic-resistance data are analysed over varying geographical scales, such as through surveillance systems, significant differences in the antibioticresistance profiles of bacterial isolates are often detected ${ }^{37,38}$. Changes in resistance patterns over time are also often detected, but these changes are not always reflective of obvious changes in antibiotic usage ${ }^{5,7,22}$. A major challenge in this sort of study is to correctly characterize exposure to antibiotics and any other factor that might select for changes in antibiotic resistance. When factors that can influence the outcome of a study are not accounted for in the design or analysis, the estimated effects and interpretations will be biased.

Consequently, one of the most difficult aspects of ecological studies of antibiotic resistance is the selection of appropriate comparison (or control) groups ${ }^{2,39}$ because this requires knowledge of the potential causes of antibiotic resistance. For example, farms that do not use antibiotics are often selected as controls because they are assumed to be devoid of the selection pressures provided by antibiotic use. Why, then, do we find antibiotic resistance on farms that do not use antibiotics? How severe are the biases when we compare farms that use antibiotics with farms that do not use antibiotics, when antibiotic resistance can be found on all of them? The topic of control-group selection in ecological studies of antimicrobial resistance has received little attention, but in hospital studies, control-group selection has been shown to have an impact on the apparent relationship between antibiotic use and resistance ${ }^{40}$. In addition, the choice of control groups can vary depending on the specific question being addressed in the study ${ }^{41}$.

The eighteenth century Scottish philosopher David Hume wrote “... we may define a cause to be an object followed by another ... where, if the first object had not been, the second never had existed." This idea has since been referred to as counterfactual causality because the only study design that can assess accurately the existence of a causal relationship and the strength of the association between cause and effect is one in which a population is simultaneously exposed and unexposed to the cause $\mathrm{e}^{42-44}$. Only one of these two exposures can actually occur in the target population. This is termed the factual (or actual) exposure. The other exposure is a hypothetical alternative to the actual exposure, and it is termed the counterfactual exposure. The outcome experience of the target population can obviously be observed only under the factual exposure; the outcome experience of the target population under the counterfactual exposure is termed the counterfactual outcome experience. 
In practice, the causal relationship is approximated by replacing the counterfactual outcome experience of the target population with the outcome experience of a substitute group that experiences the exposure that is counterfactual for the target population. This substitute group is often called the control or comparison group. For example, instead of studying a group of farms that simultaneously receives and does not receive antibiotics (the counterfactual model of causation), we select some farms that use antibiotics and compare these farms with others that do not use antibiotics to estimate the relationship between antibiotic use and resistance. As the difference between the counterfactual outcome experience of the target group and the actual outcome of the substitute group increase, the degree of confounding and introduced bias also increases. All studies that use a comparison group suffer from this assumption that the outcome experience of the comparison group resembles the outcome experience the target group would have experienced under the counterfactual exposure; however, in studies involving complex environments with many potential causes, the ability to quantify the causal relationship accurately becomes extremely difficult.

Confounding bias is introduced when the substitute group (in the case of the example used previously, farms that do not use the antibiotic) is affected to a greater or lesser extent than the target group by another factor that is also capable of causing changes in resistance levels. In other words, confounding bias is introduced when there is an uneven distribution of the alternative cause between those farms that use the antibiotic and those farms that do not. No substitute group will ever be a perfect match for the target group, and therefore, there will always be some level of bias regardless of the study design or the analytical methods used ${ }^{44,45}$. The goal, then, is to collect as much information about potential alternative causes of the outcome of interest in order to design the most accurate studies and to control for the presence of the remaining confounding variables in the analysis. In the case of antibiotic resistance, ecological studies should take into consideration all of the important potential factors that can affect the emergence, dissemination and persistence of resistance. This can seem to be a daunting task, but we believe that the methods of landscape ecology can help overcome some of this confounding bias.
Selection pressures - a complex array To understand the reasons for the presence of antibiotic resistance and to predict future changes in resistance, it is necessary to understand and quantify the distributions of as many of the factors that select for resistance genes and resistant organisms as possible. Obviously the use of an antibiotic that directly selects for resistance is a significant factor. There are many other factors that can also select for the same resistance, and therefore, there can be causes for observed resistance levels that might not be related to antibiotic use. To build the most accurate predictive model possible, the relative importance of each of these causal factors should be estimated. Theoretically, the selection and persistence of an antibioticresistance gene that has been introduced into the environment might have more to do with the presence of additional genes that confer resistance to chemicals and metals or that provide an ecological fitness advantage to the cell, or with alternative sources of the antibiotic than to the presence of a primary antibiotic-selection pressure ${ }^{46}$.

\section{....in studies involving complex environments ... the ability to quantify the causal relationship accurately becomes extremely difficult...}

In this paper we use a hypothetical agricultural ecosystem to explore the relationship between antibiotic use in animal agriculture and antibiotic resistance. The landscape depicted in the relief map in FIG. 1 a will be used to demonstrate how a model that addresses the complex relationship between antibiotic use and resistance can be built. FIGURE $1 \mathrm{~b}$ shows the farms that do and do not use antibiotic 1 . The common expectation is that resistance to antibiotic 1 will be higher on farms that use antibiotic 1 . In BOX 1 , the relationship between the strength of the selection pressure exerted by antibiotic 1 and the probability of finding resistance on farms that use this antibiotic compared with those that do not is examined. To begin this simple hypothetical scenario, the only pressure selecting for resistance to antibiotic 1 is the use of antibiotic 1, and this ideal situation is termed the 'baseline' scenario. As the strength of the selection pressure increases, the probability of finding higher levels of resistance on farms that use the antibiotic also increases.
Consider a hypothetical scenario of a genetic linkage between two genes that confer resistance to two unrelated antibiotics (antibiotic 1 and antibiotic 2). When antibiotic 2 is used, the genes conferring resistance to both antibiotics 1 and 2 are under a positive selection pressure because they are genetically linked in the bacterium. If the use of antibiotic 2, a competing cause of resistance to antibiotic 1 , is not evenly distributed among the sampled units or is not controlled for in the analysis, we are likely to make a biased prediction of the association between the use of antibiotic 1 and resistance to this antibiotic. For example, FIG. $1 \mathrm{~b}$ shows the farms that use antibiotic 1 (farms represented by brown cows) and those that do not (farms represented by white cows). Assume that the farms in white use antibiotic 2 instead of antibiotic 1 to control the same disease. An example of this would include the different antibiotic classes available to treat bovine respiratory disease or bovine calf scours. When comparing farms that use antibiotic 1 with those that do not, we would expect resistance to antibiotic 1 to be higher on the farms that use this antibiotic than on farms that use antibiotic 2 . However, because of the genetic linkage, we might see very little difference in resistance to antibiotic 1 between the two groups because the second group of farms is using an antibiotic that selects for the same resistance (BOX 1). As the strength of the selection pressure imposed by antibiotic 2 increases, and the frequency of the linkage increases, the probability of finding a difference between farms that use antibiotic 1 compared with those that do not diminishes.

Many examples of this effect have been described. For example, as some countries have reduced the amount of antibiotic used in animal agriculture, specific resistance has persisted in the apparent absence of primary selection pressure ${ }^{5,722}$. A more thorough genetic analysis of these isolates reveals that linkages to other antibiotics ${ }^{5,47}$ can partially explain this persistence. Commonly used disinfectants can also help explain certain patterns of antimicrobial resistance. For example, quaternary ammonium compounds (QAC) are commonly used in animal production, and resistance can develop to these disinfectants ${ }^{48,49}$. There are now many examples of QACresistance genes being linked genetically to antimicrobial-resistance genes ${ }^{50,51}$. The QAC-resistance gene is often located on a plasmid, increasing the potential for horizontal gene transfer among bacteria. In addition, a QAC-resistance gene cassette 
has been found in class I integrons in various bacteria, including Salmonella spp. ${ }^{52}$ Consequently, the use of antibiotics or disinfectants is one way to select for antibiotic resistance in the absence of primary selection pressure.

Although bacteria can require metals for survival, high levels can be toxic to the cell, and therefore many bacteria possess mechanisms of resistance to specific metals. Evidence of co-selection of metal and antibiotic resistance is increasingly being observed $^{53,54}$. The metals that can co-select for antibiotic resistance often have heterogeneous distributions in the environment, therefore differentially affecting bacterial populations at different sites (FIG. 1c). Mercury is commonly found in the environment owing to its widespread use in various industries and in dentistry. Mercury-resistance genes can be found in association with mobile genetic elements in bacteria ${ }^{55}$, and these elements commonly possess antibiotic-resistance genes ${ }^{56}$. For example, the transposon $\operatorname{Tn} 21$ possesses the mercury resistance (mer) operon and a class 1 integron that often includes antibioticresistance genes ${ }^{52,57,58}$.
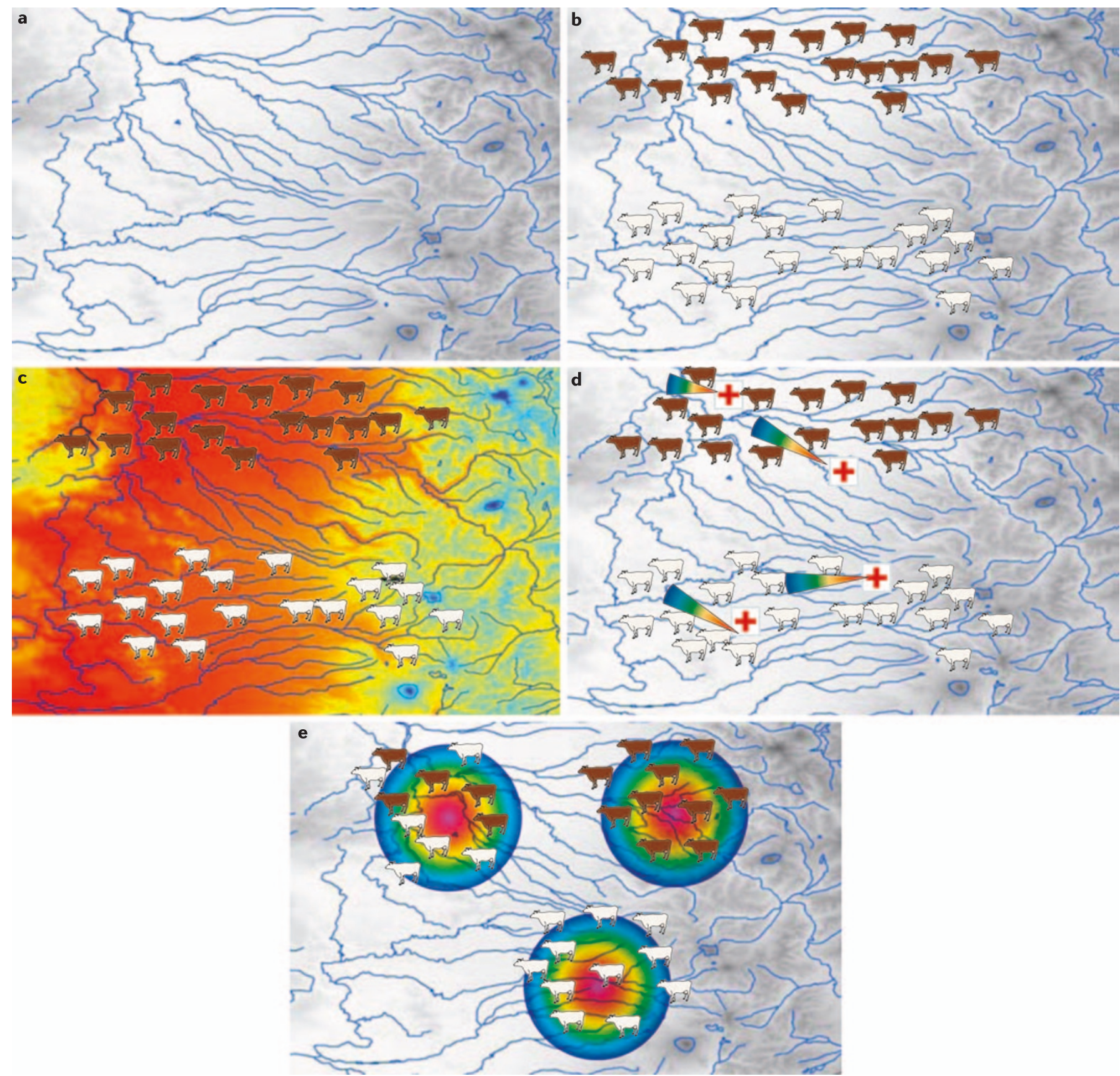

Figure 1 | A hypothetical agricultural ecosystem to explore the relationship between antibiotic use in animal agriculture and antibiotic resistance. a|Baseline relief map showing the topography of the landscape and waterway routes. $\mathbf{b}$ | The distribution of farms that use antibiotic 1 (brown cows) and farms that do not use antibiotic 1 (white cows). c|The concentration of a metal, such as copper, throughout the landscape. Red areas have high concentration of copper and blue areas have lower concentrations. d | The location of hospitals on the waterways. Antibiotics and resistant bacteria are disseminated in effluent in a plume effect, with higher levels nearer the source. e| The potential spread of resistant bacteria to nearby farms by airborne dissemination or movement of bacteria on fomites. The spatial autocorrelation among farms is demonstrated by this linkage. 
Copper is another metal that is toxic to many bacteria at high levels. Copper is commonly used in the production of animals for food and as a bactericide and fungicide in horticulture. It can also be found in the environment naturally or as a result of agricultural runoff. Copper has been shown to select for copper-resistant bacterial isolates, and in one study, isolates from copper-amended fields were more resistant to copper and antibiotics than strains from control fields ${ }^{59}$. A recent study of Enterococcus faecium from broiler and turkey farms demonstrated the presence of copper resistance conferred by the transferable copper-resistance gene $\operatorname{tcr} B$. Of concern was the finding that the $\operatorname{tcr} B$ gene was linked to the vatE gene on the same plasmid. The vat $E$ gene encodes an acetyltransferase that can inactivate the antibiotic virginiamycin, thereby enabling a copper selection pressure to maintain virginiamycin resistance, even in the absence of virginiamycin use ${ }^{60}$. Finally, a relationship between $t c r B$, glycopeptide resistance and macrolide resistance was observed in E. faecium isolated from pigs in Denmark ${ }^{61}$; in this case, the continued use of copper sulphate in swine production did not seem to maintain the combined resistance to copper, glycopeptides and macrolides over time, suggesting that the resistance genes might not be directly linked to metal resistance in all cases.

Environmental selection pressures exist in the form of antibiotics and their metabolites. Given that certain microorganisms, especially those that live predominantly in the soil, are capable of producing antibiotics, increased levels of resistance would be expected in areas where these antibioticproducing organisms are in abundance ${ }^{13}$. However, antibiotics can also be found in the waterways and in soil ${ }^{18,62}$ after release from hospitals, sewage-treatment plants, factories, runoff from agricultural facilities and runoff and leaching from agricultural manure applied to fields ${ }^{17,62,63}$. These antibiotics are potentially capable of exerting a selection pressure. As the antibiotic is released, there will often be a concentration gradient, with the highest levels of antibiotic occurring closest to the source. In the example depicted in FIG. 1d, hospitals are shown releasing their treated wastewater into rivers, a common scenario in many rural areas around the world. Farms from both antibiotic groups are exposed to this effluent, with farms nearest the source under the highest selection pressure. Depending on the

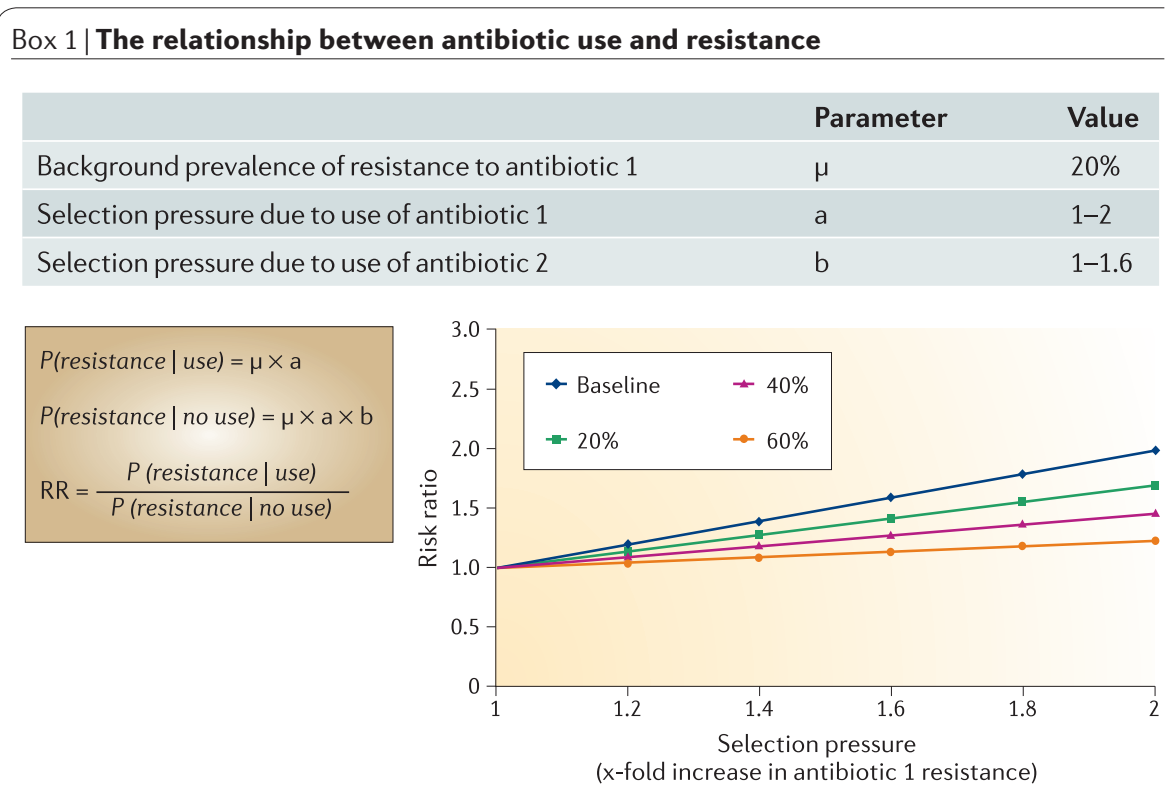

In this model, the probability $(P)$ of finding resistance to antibiotic 1 on farms that use antibiotic 1 , $P$ (resistance / use), is compared with the probability of finding resistance on those farms that do not use antibiotic 1 but use antibiotic 2 instead, $P$ (resistance / no use). The comparison between the two groups is expressed as a risk ratio (RR), and the null value for the $R R$ (no difference between the groups) equals 1 . The selection pressure exerted by antibiotic 1 results in an $x$-fold increase in the amount of resistance to antibiotic 1 (ranging from one to twofold in this example), and a selection pressure of 1 implies no selective effect. The selection pressure of antibiotic 2 results in an $\times$-fold change in resistance to antibiotic 1 by genetic linkage of the relevant resistance genes and varies between $0-60 \%$ of the pressure exerted by antibiotic 1 . The background prevalence of resistance to antibiotic 1 is set at $20 \%$. The baseline represents the true causal effect of the use of antibiotic 1 on resistance to antibiotic 1 , and the other three scenarios represent an increasing influence of antibiotic 2 on resistance to antibiotic 1 (see figure).

spatial distribution of farms, the apparent relationship between the use of antibiotic 1 and resistance to antibiotic 1 can be both overestimated and underestimated, with the bias becoming stronger as the influence of these environmental sources increases and as the geographical distribution of farms that use and do not use the antibiotic becomes more heterogeneous with respect to the environmental levels of these competing causes. The challenge associated with incorporating this information into prediction models of antibiotic resistance is to estimate the fate of these antibiotics in the environment. Some antibiotics bind more strongly to soil than others, and this strength can vary with soil type ${ }^{64-66}$. In addition, the antibiotics that are released into the environment can be taken up by growing plants or crops, again making it difficult to model the fate and potential activity of these antibiotics in the environment ${ }^{67}$.

\section{Routes of transmission}

Perhaps the most difficult aspect of the design of antibiotic-resistance studies is the inclusion of the routes and probabilities of transmission of the antibiotic-resistant organisms. Bacteria can be disseminated between sites, and therefore resistance can be found in areas irrespective of any selection pressures. Transmission is a source of new resistance genes, not just the potential amplification of existing genes owing to proximate selection pressures ${ }^{68}$. When considering the effects of antibiotic use, indirect effects (which include transmission) might be the most important contributors to the emergence and dissemination of antibiotic resistance at the ecological level ${ }^{39}$.

As an example of the potential impact of transmission on antibiotic-resistance studies, consider the effluent and surface-water runoff example shown in FIG. $1 \mathrm{~d}$. In this example, we can assume that hospital effluent contains antibiotic-resistant organisms in addition to antibiotics ${ }^{68,69}$. Farms that are closer to the source of the effluent have a higher probability of acquiring organisms from this source. Bacteria also frequently move from agricultural facilities and from manure applications on fields, thereby 
spreading resistance genes into the environment ${ }^{34,62,70,71}$. To accurately predict how microorganisms will be disseminated by water, additional hydrological and geophysical properties must be considered, including the frequency, duration, and intensity of rainfall, slope, vegetative cover, soil type, organic-matter content, temperature, available nutrients and the presence of toxic materials, to name just a few ${ }^{72}$. In the case of livestock systems, an additional consideration is the movement of animals between farms.

In ecological studies, additional routes such as the airborne dissemination of resistant bacteria ${ }^{73,74}$, as well as hospital admissions and people returning to the community after hospital stays ${ }^{39}$, might be significant. Organisms can be moved from site to site, and potentially over large geographical distances, by mechanical transmission on fomites or other mechanical vectors, or through the movement of biological vectors. FIGURE 1e depicts this scenario for the farm example, where all farms located within a certain radius share common bacteria. The result will be similar to the result described previously, in which the effect of antibiotic use on resistance is masked by the overall transmission of resistance among farms. Little research has been conducted on how to control our analyses for this type of transmission. However, as we will describe, statistical analyses and mathematical models can be designed to account for this spatial dependency.

\section{Incorporating landscape ecology}

As described previously, many factors can influence the distribution of antibioticresistant bacteria in the environment. Because of the many potential competing causes for changes in antibiotic-resistance patterns, establishing an accurate relationship between antibiotic use and resistance is challenging.

The tools of landscape ecology include methods for capturing complex spatial data, for incorporating new types of explanatory data, and for analysing these data using spatial statistics and mathematical models ${ }^{75,76}$. One of the fundamental tools is the geographical information system (GIS), a combination of hardware, software and analytical methods for relating data on varying spatial scales. Spatial data can exist as points, such as the location of sampling; as lines, such as rivers and roads; or as areas, such as lakes and census tracts. One derived data type is raster - a continuous surface of pixels, in which each pixel has an estimated value for each variable of interest. Each variable is included as a separate layer or theme in the GIS and can be overlaid with other variables to view and analyse their spatial relationships. A second tool of landscape ecology is to incorporate spatial data on features, which are measured by a sensor that is distant from the study site, a technique known as remote sensing. For example, data obtained from satellite imagery or aerial photographs can be included in the GIS, providing data about variables that previously were difficult or impossible to obtain and analyse. These data can include soil types, land cover, elevation data, surface temperatures and vegetation moisture. FIGURE $1 \mathrm{~b}$ demonstrates the method of overlaying variables and comprises three data layers: point data of the farm location, line data of the rivers, and a continuous surface of elevation that has been derived from remotely sensed satellite imagery.

Finally, there are methods for transforming and analysing these spatial data. For example, there are many approaches to interpolating the values of variables at sites where data were not obtained. The result is a continuous surface of data for that variable. A map such as that shown in FIG. 1c depicts the same farms as in FIG. 1 b, but now the concentration of a metal such as copper has been incorporated. It is assumed that the copper measurements are taken at a finite number of locations, and estimates of copper concentrations across the landscape are obtained through these interpolation techniques. In this example, we are assuming that copper is capable of selecting for antibiotic-resistant organisms, and therefore, an analysis that fails to adjust for the distribution of copper will make biased conclusions about the relationship between the use of that antibiotic and resistance to it. The inclusion of a surface map of environmental copper concentrations in the GIS can help explain the observed levels of resistance across the landscape by controlling for its effect in the analysis.

There are many types of questions that can be addressed by combining the GIS, spatial statistics and mathematical models. Some examples include: is there spatial clustering of resistance and, if so, what factors are associated with this clustering? What areas would be predicted to be high-risk areas for the introduction of antibiotic-resistant organisms, given the selection pressures that exist at that site? How did the resistance determinants spread through the environment? How long will it take for the resistant organisms or resistance genes to diffuse through the studied ecosystem? An output of this type of analysis might be a risk map that has been used to document the geographical, seasonal or annual occurrence of infectious agents, especially vector-borne diseases ${ }^{33,77-}$ ${ }^{80}$. Analyses involving diarrhoeal diseases have also been conducted, and some of these have included socioeconomic and sanitation variables ${ }^{81-85}$.

Spatial analyses and landscape ecology approaches have rarely been incorporated into ecological investigations of antibiotic resistance. As an example of how this type of analysis could be used in antibioticresistance studies, suppose that the goal of a project were to determine the factors associated with increased resistance in environmental isolates of Escherichia coli. The first step might be to determine the spatial characteristics of the area in proximity to each sampling point using the GIS database $\mathrm{e}^{75,76}$. Sites can be characterized in terms of their elevation, proximity to a river or stream, proximity to other potential contamination sources such as hospitals, clinics and agricultural premises, soil type, organic matter content and vegetation characteristics. A 'buffer' analysis might follow, in which additional variables are created that quantify the characteristics of the area surrounding each sampling point (the 'buffer'). For example, it is possible to determine whether any of the resistant isolates were found within a specified radius of a specific feature, such as a hospital or an area containing high levels of copper and mercury. Finally, prediction maps and mathematical models can be constructed using statistical analyses that include these new spatial data and account for the hierarchical nature of the data. The end result is a prediction of antibiotic-resistance dynamics in E. coli that has been adjusted for the specific environmental features in the landscape being studied.

\section{How to address spatial heterogeneity}

There is one key concept that underlies all of the previous examples: the selection pressures and transmission probabilities at one site affect those at neighbouring sites. This is the basic definition of spatial autocorrelation, which refers to the principle that objects located closer in space are more likely to be similar to each other than to objects that are located further apart. This principle is known as the first law of geography $^{86}$. Spatial dependence is crucial in the argument for microbial biogeography ${ }^{29}$, 
in which the diversity and abundance of the microbial community is non-random. We would therefore expect a positive spatial autocorrelation between genetic distance and geographical distance (that is, the closer two organisms are in space, the more likely they are to be genetically similar, and vice versa).

Based on the distribution of selection pressures in the environment as well as the potential routes of transmission discussed previously, there is likely to be a high degree of spatial autocorrelation with respect to antibiotic resistance. In the examples above, farms located closer together (or within the same network) will probably share common selection pressures and will be more likely to share common resistant bacteria and resistance genes. For example, if bacteria are being disseminated in rivers, then farms upstream will affect those downstream, regardless of which antibiotics are being used on an individual farm.

In the example shown in FIG. 1e, farms that use and do not use antibiotic 1 are interspersed, lacking the geographical separation shown in FIG. 1b. Without considering spatial location, the farms will seem to be more similar to each other, thereby diluting the measurable effect that antibiotic use has on resistance. The tools of landscape ecology can help adjust analyses for the existence of this spatial autocorrelation ${ }^{87-89}$. For example, statistical analyses can account for the characteristics of the farms that are nearest to each study farm.

There are two alternative methods for dealing with spatial heterogeneity in antibiotic-resistance studies. Prospective longitudinal studies can be designed in which the presence of environmental antibiotic resistance and other causes of resistance, such as metals, disinfectants and natural environmental antibiotics, at sites is known a priori and is assumed not to change over the study period. Study subjects (such as farms) can then be matched to remove the effect of these factors on the measured relationship between antibiotic use and resistance. However, this type of study design is not generally feasible owing to the difficulty in measuring each of these confounding factors at a fine geographical scale.

Perhaps more important is our lack of knowledge of all the important causes of antibiotic resistance on a spatial scale. The usual study design is to select groups, either prospectively or retrospectively, based on antibiotic use and measure the presence and level of antibiotic resistance. Because in this study design the presence

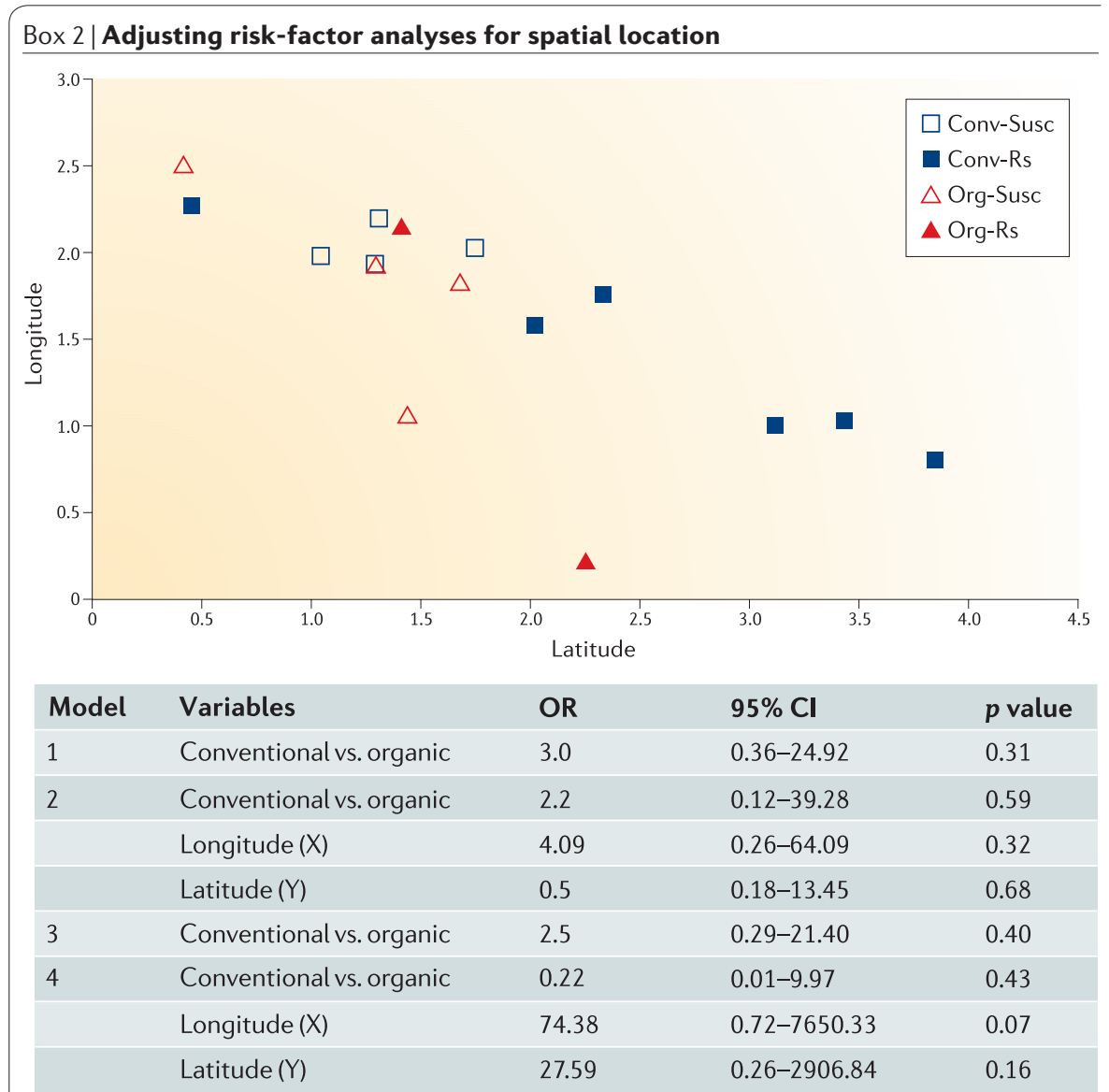

The odds of finding sulphadimethoxine-resistant Shiga-toxin producing Escherichia coli (STEC) on conventional dairy farms compared with organic dairy farms is assessed from data collected in Minnesota. For this simple example, only the first STEC isolate from a given farm is used in the analysis. The figure depicts the spatial location of each isolate, resistant (Rs) and susceptible (Susc), from both conventional (Conv) and organic (Org) dairies. The exact latitude and longitude coordinates have been normalized so that the precise location of each dairy cannot be determined. A logistic regression model is used to compare the odds of finding resistant STEC in conventional dairies compared with organic dairies; $95 \%$ confidence intervals $(\mathrm{Cl})$ are given. In model 1 , the only variable included in the model is farm type, comparing organic versus conventional. In model 2 , the latitude and longitude coordinates for each farm are included. The odds ratio (OR) for farm type decreases from 3.0 to 2.2, indicating a spatial relationship of STEC, regardless of farm type. In model 3 , the conventional farm with resistant STEC, located at approximately 0.5 latitude and 2.3 longitude, is removed from the analysis, as an example of the potential importance of spatial outliers in field-conducted research of antibiotic resistance and their impact on study conclusions. The OR for farm type in this model is 2.5 . In model 4 , the same outlying conventional farm is removed from the analysis and the OR is also adjusted for spatial location and decreases to 0.22 . This implies that without considering and including spatial location in the analysis, conventional farms seem to be a risk factor for possessing resistant STEC. After adjusting for the farm locations, organic farms seem to be at a higher risk of having resistant STEC. It is important to note that this example is for demonstration purposes only. The data probably violate some of the assumptions of the logistic regression model, including the assumption of linearity in the $\ln (\mathrm{Odds})$. Furthermore, the coefficients are all non-significant, owing in large part to the small sample size of this example dataset.

of numerous potential causes of resistance are unknown, these potential confounders of the relationship between antibiotic use and resistance must be controlled analytically. A simple method of assessing the importance of spatial autocorrelation is to analyse the data with and without taking spatial location into account. If effect measures (for example, the odds ratio) differ substantially between the two models, then the spatial nature of the data must be included to reduce bias. Including a spatial component in analyses will control for a range of factors that have 
a spatial distribution, although the relationship between specific factors and the outcome of interest cannot be investigated. If logistic regression is used to analyse case-control data, the $\mathrm{X}$ and $\mathrm{Y}$ coordinates of the sampling points can be forced into the model and the change in the odds ratios of the risk factors of interest (usually the estimate associated with antibiotic use) can be assessed. More complex methods are available, such as Bayesian logistic regression models that incorporate spatial autocorrelation.

As an example, consider the data presented in BOX 2 in which dairy farms in Minnesota were sampled for Shiga toxinproducing E. coli (STEC). In the dataset presented, 16 farms were positive for STEC, and sulphadimethoxine resistance was assessed for each isolate. Because the farms were either conventional (using antibiotics) or organic (no antibiotics), the farm type was investigated as a potential risk factor for sulphadimethoxine-resistant STEC. As seen in BOX 2, the estimate of the effect of the farm type on resistance was reduced when spatial location was included. Furthermore, when one conventional farm was removed from the model, organic farms became a risk factor for sulphadimethoxine-resistant STEC. In this example, therefore, the estimated relationship between antibiotic use and resistance is biased if spatial location is not taken into account.

As discussed previously, there is considerable antibiotic resistance in the environment, even in areas that seem to be devoid of anthropogenic selection pressures. This background resistance would seem to be a constant nuisance in our attempts to relate specific selection pressures and antibiotic resistance in ecological settings. Fortunately, many of the statistical analyses and mathematical models that are available, including those that incorporate spatial attributes, can adjust the estimated relationships for this background. In most standard regression models, the intercept term is used to represent the background level of the outcome variable that cannot be explained by the predictor variables included in the model. In this crude manner, the intercept term is adjusting the relationship between the independent and dependent variables for the background level of resistance. In more complex models, specific terms can be added, for example as random effects, that serve to 'absorb' the variation in the outcome variable that cannot be explained by the predictor variables. As an example, suppose that the background level of resistance varies over geographical regions, but that we do not have data to explain why this variation exists. The statistical models that are built can incorporate a random effect term for 'region', which explains the variation in resistance that might be attributable to differences among regions but which is not explained by the other variables in the model. Again, this approach helps adjust our relationships for background levels of resistance that exist for reasons that we cannot explain.

Finally, another important consideration when analysing antibiotic use and resistance data relates to the issue of multiscale variation, also known as scaling. The causal factors that are important in the emergence, dissemination and persistence of antibiotic resistance might have a spatial relevance at different scales. As discussed in a recent paper, antibiotic use can be regarded as primarily a local phenomenon as medications are administered at the individual or population level ${ }^{90}$. However, veterinarians serve many farms in an area, and therefore, all farms served by the same veterinary practice might be under similar selection pressures. The farms served by many different veterinary practices might all be in the same watershed, thereby increasing the spatial autocorrelation among these farms. Therefore, a population is not merely a sum of its parts but can have characteristics at each hierarchical level ${ }^{91,92}$.

As an example, a study of antibiotic resistance in bacteria isolated from cattle pneumonia cases in California found significant spatial clustering of antibiotic resistance at local and regional scales ${ }^{87}$. This could reflect the influence of the local veterinarian, the environment or any other factor that operates at these spatial scales. Variables could be included in multi-level statistical models for each of these spatial scales, similar to the random effect models described previously. These models can be designed using the data from the GIS to account for a hierarchy (for example, sites within an area, areas within a larger region). In addition, mathematical models that incorporate the data from the GIS can account for the multi-scale variation.

\section{Future directions}

In ecological studies of antibiotic resistance, there has potentially been too much focus on resistant organisms and not enough on resistance genes. The ability of bacteria to transfer resistance genes, even among distantly related bacteria, has the potential to confound all of our simple study designs ${ }^{93-95}$. Ecological studies are therefore in desperate need of methods that can assess the genomic structure of entire bacterial communities at individual sites and then relate this information to the ecological processes that exist at that site. Consequently, analyses of antibiotic-resistance emergence, dissemination and persistence might be better conducted at the level of the gene. The tools of landscape ecology would be a considerable help to the design, implementation and analysis of this type of investigation.

The emerging field of metagenomics, which assesses the genomic composition of bacterial communities in complex samples, is being used more frequently to fill this data gap ${ }^{96}$. In particular, metagenomic studies that evaluate the diversity of resistance genes ${ }^{97}$ in samples have been conducted, and a recent survey of resistance diversity in soil labelled this bacterial community the 'resistome'13. To make these studies useful in evaluating the more general potential causes of antibiotic-resistance levels, additional data from these ecosystems, such as hydrological characteristics, soil attributes and temperature distributions, will be required. Some of the studies that have analysed the microbial community at different spatial scales are discussed in a recent review paper $^{29}$. For example some studies have begun to characterize the microgeographical diversity of bacterial communities in relation to soil characteristics ${ }^{98}$. As shown in this paper, the metagenomic data at each site sampled in a study can be added to the spatial data analysis. In this manner, the factors that influence the transmission and selection of resistance can be related to the genetic diversity of bacterial populations over large spatial scales. Given the extreme heterogeneity observed in bacterial population studies that are conducted in small microgeographical soil plots, it is unclear if studies conducted over large geographical areas will provide additional information about the dynamics of antibiotic resistance.

Another type of dataset that might benefit from landscape ecology analyses is quantitative data on the resistance gene loads in samples ${ }^{34,99,100}$. Currently, resistance in a sample is based on the cultivation of specific bacteria, and categorizing the bacterial isolates into resistant and susceptible groups. If only one colony is analysed per sample, as is done in some surveillance systems ${ }^{5,38,101}$, then this is equivalent to saying that the entire sample is resistant or susceptible. However, all of the bacteria in a sample or at a given site can be under the same set of selection pressures, and therefore, the 'amount' of a resistance gene present in all of the bacteria in a sample might be a better predictor of the selection pressures being applied 
to that sample over short- and long-term timescales. In this case, the analyses would be redesigned with the load of specific resistance genes as the outcome variable. To make this approach viable, however, the laboratory methods for quantifying resistance genes in complex samples must be improved so that they are accurate for low copy number genes that are present in samples with large amounts of bacterial DNA.

\section{Conclusion}

It is commonly believed that resistance will decrease when the antibiotic is removed. Much of this assumption is based on the expected fitness cost of maintaining the resistance gene(s). Recent studies indicate that the maintenance of resistance might not impose a significant fitness $\operatorname{cost}^{23-26}$ or that this cost can be overcome ${ }^{19-21,27}$. However, there is also a large background pool of resistance, probably a result of the fact that many resistance genes protect the bacterial cell in such a way that they are likely to be functional against other compounds in the environment. It should therefore not be surprising that these resistance genes are found in 'pristine' areas that lack anthropogenic disturbance. Many of these genes are ancient on an evolutionary timescale and have perhaps been modified to protect the cell against human-produced antibiotic compounds. It is also possible that we are not accurately measuring the diversity of selection pressures and routes of transmission that are influencing the evolution, dissemination and persistence of antibiotic resistance. Landscape ecology provides a framework for understanding these heterogeneous pressures and transmission probabilities. Through improvements in study design, exposure classification and data analysis, the tools of landscape ecology should aid in a more accurate assessment of this complex problem of antibiotic resistance.

Randall S. Singer and George Maldonado are at the Division of Environmental Health Sciences, School of Public Health, University of Minnesota, Minneapolis, Minnesota 55455 USA.

Randall S. Singer is also at the following: the Department of Veterinary and Biomedical Sciences, University of Minnesota, St. Paul, Minnesota 55108 USA; the Instituto de Medicina Preventiva Veterinaria, Facultad de Ciencias Veterinarias, Universidad Austral de Chile, Valdivia, Chile; and the Departamento de Microbiología, Facultad de Ciencias Biológicas, Universidad de Concepción, Concepción, Chile.

Michael P. Ward is at the Department of Veterinary Integrative Biosciences, Texas A\&M University, College Station, Texas 77843-4458 USA. Correspondence to R.S.S. e-mail: singe024@umn.edu doi:10.1038/nrmicro1553
1. Salyers, A. A. \& Amabile-Cuevas, C. F. Why are antibiotic resistance genes so resistant to elimination? Antimicrob. Agents Chemother. 41, 2321-2325 (1997)

2. Salyers, A. A., Shoemaker, N. B. \& Bonheyo, G. T. in Bacterial Resistance to Antimicrobials (eds Lewis, K. Salyers, A. A., Taber, H. W. \& Wax, R. G.) 1-18 (Marcel Dekker, New York, 2002).

3. Summers, A. O. Generally overlooked fundamentals of bacterial genetics and ecology. Clin. Infect. Dis. 34, (Suppl. 3), S85-S92 (2002).

4. Aarestrup, F. M., Kruse, H., Tast, E., Hammerum, A M. \& Jensen, L. B. Associations between the use of antimicrobial agents for growth promotion and the occurrence of resistance among Enterococcus faecium from broilers and pigs in Denmark, Finland, and Norway. Microb. Drug Resist. 6, 63-70 (2000)

5. Aarestrup, F. M. et al. Effect of abolishment of the use of antimicrobial agents for growth promotion on occurrence of antimicrobial resistance in fecal enterococci from food animals in Denmark. Antimicrob. Agents Chemother. 45, 2054-2059 (2001).

6. Casewell, M., Friis, C., Marco, E., McMullin, P. \& Phillips, I. The European ban on growth-promoting antibiotics and emerging consequences for human and animal health. J. Antimicrob. Chemother. 52, 159-161 (2003).

7. Heuer, O. E., Pedersen, K., Andersen, J. S. \& Madsen, M. Vancomycin-resistant enterococci (VRE) in broiler flocks 5 years after the avoparcin ban. Microb. Drug Resist. 8, 133-138 (2002).

8. Heuer, O. E., Pedersen, K., Jensen, L. B., Madsen, M. \& Olsen, J. E. Persistence of vancomycin-resistant enterococci (VRE) in broiler houses after the avoparcin ban. Microb. Drug Resist. 8, 355-361 (2002)

9. Phillips, I. et al. Does the use of antibiotics in food animals pose a risk to human health? A critical review of published data. J. Antimicrob. Chemother 53, 28-52 (2004).

10. Kieser, T., Bibb, M. J., Buttner, M. J., Chater, K. F. \& Hopwood, D. A. Practical Streptomyces Genetics (John Innes Centre, Norwich, UK, 2000)

1. Waksman, S. The role of antibiotics in nature. Perspect. Biol. Med. 4, 271-287 (1961).

12. Yim, G., Wang, H. H. \& Davies, J. The truth about antibiotics. Int. J. Med. Microbiol. 296, 163-170 (2006)

13. D'Costa, V. M., McGrann, K. M., Hughes, D. W. \& Wright, G. D. Sampling the antibiotic resistome. Science 311, 374-377 (2006).

14. Davies, J. Are antibiotics naturally antibiotics? J. Ind. Microbiol. Biotechnol. 33, 496-499 (2006)

15. Schmitt, H., Stoob, K., Hamscher, G., Smit, E. \& Seinen, W. Tetracyclines and tetracycline resistance in agricultural soils: microcosm and field studies. Microb. Ecol. 51, 267-276 (2006)

16. Kummerer, K. Drugs in the environment: emission of drugs, diagnostic aids and disinfectants into wastewater by hospitals in relation to other sources - a review. Chemosphere 45, 957-969 (2001).

17. Kummerer, K. \& Henninger, A. Promoting resistance by the emission of antibiotics from hospitals and households into effluent. Clin. Microbiol. Infect. 9 , 1203-1214 (2003).

18. Kolpin, D. W. et al. Pharmaceuticals, hormones, and other organic wastewater contaminants in U.S. streams, 1999-2000: a national reconnaissance. Environ. Sci. Technol. 36 1202-1211 (2002)

19. Schrag, S. J., Perrot, V. \& Levin, B. R. Adaptation to the fitness costs of antibiotic resistance in Escherichia coli. Proc. Biol. Sci. 264, 1287-1291 (1997).

20. Andersson, D. I. \& Levin, B. R. The biological cost of antibiotic resistance. Curr. Opin. Microbiol. 2, 489-493 (1999).

21. Lenski, R. E. Bacterial evolution and the cost of antibiotic resistance. Int. Microbiol. 1, 265-270 (1998)

22. Sorum, M. et al. Prevalence, persistence, and molecular characterization of glycopeptide-resistant enterococci in Norwegian poultry and poultry farmers 3 to 8 years after the ban on avoparcin. Appl. Environ. Microbiol. 72, 516-521 (2006)
23 Khachatryan, A. R., Hancock, D. D., Besser, T. E. \& Call, D. R. Role of calf-adapted Escherichia coli in maintenance of antimicrobial drug resistance in dairy calves. Appl. Environ. Microbiol. 70, 752-757 (2004).

24. Khachatryan, A. R., Hancock, D. D., Besser, T. E. \& Call, D. R. Antimicrobial drug resistance genes do not convey a secondary fitness advantage to calfadapted Escherichia coli. Appl. Environ. Microbiol. 72, 443-448 (2006)

25. Luo, N., Sahin, O., Lin, J., Michel, L. O. \& Zhang, Q. In vivo selection of Campylobacter isolates with high levels of fluoroquinolone resistance associated with gyrA mutations and the function of the CmeABC efflux pump. Antimicrob. Agents Chemother. 47, 390-394 (2003).

26. Sander, P. et al. Fitness cost of chromosomal drug resistance-conferring mutations. Antimicrob. Agents Chemother. 46, 1204-1211 (2002).

27. Levin, B. R., Perrot, V. \& Walker, N. Compensatory mutations, antibiotic resistance and the population genetics of adaptive evolution in bacteria. Genetics 154, 985-997 (2000).

28. Forman, R. T. T. Land Mosaics: The Ecology of Landscapes and Regions (Cambridge University Press, Cambridge UK, 1995).

29. Martiny, J. B. et al. Microbial biogeography: putting microorganisms on the map. Nature Rev. Microbiol. 4, 102-112 (2006).

30. Pavlovsky, E. N. Natural Nidality of Transmissible Diseases: With Special Reference to the Landscape Ecology of Zooanthroponoses. (University of Illinois Press, Urbana Illinois, 1966).

31. Kitron, U. Landscape ecology and epidemiology of vector-borne diseases: tools for spatial analysis. J. Med. Entomol. 35, 435-445 (1998).

32. Reisen, W. K., Lothrop, H. D., Presser, S. B., Hardy, J. L. \& Gordon, E. W. Landscape ecology of arboviruses in southeastern California: temporal and spatial patterns of enzootic activity in Imperial Valley, 1991-1994. J. Med. Entomol. 34, 179-188 (1997).

33. Guerra, M. et al. Predicting the risk of Lyme disease: habitat suitability for Ixodes scapularis in the north central United States. Emerg. Infect. Dis. 8 289-297 (2002).

34. Pei, R., Kim, S. C., Carlson, K. H. \& Pruden, A. Effect of river landscape on the sediment concentrations of antibiotics and corresponding antibiotic resistance genes (ARG). Water Res. 40, 2427-2435 (2006).

35. Unicomb, L. E. et al. Low-level fluoroquinolone resistance among Campylobacter jejuni isolates in Australia. Clin. Infect. Dis. 42, 1368-1374 (2006).

36. Davelos, A. L., Kinkel, L. L. \& Samac, D. A. Spatial variation in frequency and intensity of antibiotic interactions among Streptomycetes from prairie soil. Appl. Environ. Microbiol. 70, 1051-1058 (2004).

37. Swaminathan, B., Barrett, T. J. \& Fields, P. Surveillance for human Salmonella infections in the United States. J. AOAC Int. 89, 553-559 (2006).

38. Zhao, S. et al. Antimicrobial resistance and genetic relatedness among Salmonella from retail foods of animal origin: NARMS retail meat surveillance. Foodborne. Pathog. Dis. 3, 106-117 (2006).

39. Lipsitch, M. \& Samore, M. H. Antimicrobial use and antimicrobial resistance: a population perspective. Emerg. Infect. Dis. 8, 347-354 (2002).

40. Harris, A. D. et al. Control-group selection importance in studies of antimicrobial resistance: examples applied to Pseudomonas aeruginosa, Enterococci, and Escherichia coli. Clin. Infect. Dis. 34, 1558-1563 (2002).

41. Lipsitch, M. Measuring and interpreting associations between antibiotic use and penicillin resistance in Streptococcus pneumoniae. Clin. Infect. Dis. 32, 1044-1054 (2001).

42. Rubin, D. B. Estimating causal effects of treatments in randomized and nonrandomized studies. J. Educ. Psychol. 66, 688-701 (1974).

43. Hofler, M. Causal inference based on counterfactuals. BMC. Med. Res. Methodol. 5, 28 (2005).

44. Maldonado, G. \& Greenland, S. Estimating causal effects. Int. J. Epidemiol. 31, 422-429 (2002). 
45. McGowan, J. E. Jr. Antimicrobial resistance in hospital organisms and its relation to antibiotic use. Rev. Infect. Dis. 5, 1033-1048 (1983).

46. Alonso, A., Sanchez, P. \& Martinez, J. L. Environmental selection of antibiotic resistance genes. Environ. Microbiol. 3, 1-9 (2001).

47. Borgen, K., Sorum, M., Wasteson, Y., Kruse, H ¿ Oppegaard, H. Genetic linkage between erm(B) and vanA in Enterococcus hirae of poultry origin. Microb. Drug Resist. 8, 363-368 (2002).

48. Sander, J. E., Hofacre, C. L., Cheng, I. H. \& Wyatt, R. D. Investigation of resistance of bacteria from commercial poultry sources to commercial disinfectants. Avian Dis. 46, 997-1000 (2002).

49. Sidhu, M. S., Sorum, H. \& Holck, A. Resistance to quaternary ammonium compounds in food-related bacteria. Microb. Drug Resist. 8, 393-399 (2002).

50. Sidhu, M. S., Heir, E., Leegaard, T., Wiger, K. \& Holck, A. Frequency of disinfectant resistance genes and genetic linkage with $\beta$-lactamase transposon Tn552 among clinical staphylococci. Antimicrob. Agents Chemother. 46, 2797-2803 (2002).

51. Sidhu, M. S., Heir, E., Sorum, H. \& Holck, A. Genetic linkage between resistance to quaternary ammonium compounds and $\beta$-lactam antibiotics in food-related Staphylococcus spp. Microb. Drug Resist. 7 , 363-371 (2001).

52. Guerra, B. Soto S Helmuth, R \& Mendoza, M. C Characterization of a self-transferable plasmid from Salmonella enterica serotype typhimurium clinical isolates carrying two integron-borne gene cassettes together with virulence and drug resistance genes. Antimicrob. Agents Chemother. 46, 2977-2981 (2002).

53. Baker-Austin, C., Wright, M. S., Stepanauskas, R. \& McArthur, J. V. Co-selection of antibiotic and metal resistance. Trends Microbiol. 14, 176-182 (2006).

54. Stepanauskas, R. et al. Coselection for microbial resistance to metals and antibiotics in freshwater microcosms. Environ. Microbiol. 8, 1510-1514 (2006).

55. Barkay, T., Miller, S. M. \& Summers, A. O. Bacterial mercury resistance from atoms to ecosystems. FEMS Microbiol. Rev. 27, 355-384 (2003).

56. Wireman, J., Liebert, C. A., Smith, T. \& Summers, A $O$. Association of mercury resistance with antibiotic resistance in the Gram-negative fecal bacteria of primates. Appl. Environ. Microbiol. 63, 4494-4503 (1997).

57 Liebert, C. A., Hall, R. M. \& Summers, A. O. Transposon Tn21, flagship of the floating genome Microbiol. Mol. Biol. Rev. 63, 507-522 (1999).

58. Bass, L. et al. Incidence and characterization of integrons, genetic elements mediating multiple-drug resistance, in avian Escherichia coli. Antimicrob. Agents Chemother. 43, 2925-2929 (1999).

59. Berg, J., Tom-Petersen, A. \& Nybroe, O. Copper amendment of agricultural soil selects for bacterial antibiotic resistance in the field Lett. Appl. Microbiol. 40, 146-151 (2005).

60. Hasman, H. \& Aarestrup, F. M. tcrB, a gene conferring transferable copper resistance in Enterococcus faecium: occurrence, transferability, and linkage to macrolide and glycopeptide resistance. Antimicrob. Agents Chemother. 46, 1410-1416 (2002).

61. Hasman, H. \& Aarestrup, F. M. Relationship between copper, glycopeptide, and macrolide resistance among Enterococcus faecium strains isolated from pigs in Denmark between 1997 and 2003. Antimicrob. Agents Chemother. 49, 454-456 (2005).

62. Sarmah, A. K., Meyer, M. T. \& Boxall, A. B. A global perspective on the use, sales, exposure pathways, occurrence, fate and effects of veterinary antibiotics (VAs) in the environment. Chemosphere 65 , 725-759 (2006).

63. Rooklidge, S. J. Environmental antimicrobial contamination from terraccumulation and diffuse pollution pathways. Sci. Total Environ. 325, 1-13 (2004).
64. Chander, Y., Kumar, K., Goyal, S. M. \& Gupta, S. C. Antibacterial activity of soil-bound antibiotics. J. Environ. Qual. 34, 1952-1957 (2005)

65. Clay, S. A., Liu, Z., Thaler, R. \& Kennouche, H. Tylosin sorption to silty clay loam soils, swine manure, and sand. J. Environ. Sci. Health B 40, 841-850 (2005)

66. Aga, D. S. et al. Determination of the persistence of tetracycline antibiotics and their degradates in manure-amended soil using enzyme-linked immunosorbent assay and liquid chromatographymass spectrometry. J. Agric. Food Chem. $\mathbf{5 3}$ 7165-7171 (2005)

67. Kumar, K., Gupta, S. C., Baidoo, S. K., Chander, Y. \& Rosen, C. J. Antibiotic uptake by plants from soil fertilized with animal manure. J. Environ. Qual. 34 2082-2085 (2005)

68. Kummerer, K. Resistance in the environment. J. Antimicrob. Chemother. 54, 311-320 (2004).

69. Novais, C., Coque, T. M., Ferreira, H., Sousa, J. C. \& Peixe, L. Environmental contamination with vancomycin-resistant enterococci from hospital sewage in Portugal. Appl. Environ. Microbiol. 71 3364-3368 (2005).

70. Lewis, D. J. et al. Linking on-farm dairy management practices to storm-flow fecal coliform loading for California coastal watersheds. Environ. Monit. Assess. 107, 407-425 (2005)

71. Rodgers, P., Soulsby, C., Hunter, C. \& Petry, J. Spatial and temporal bacterial quality of a lowland agricultural stream in northeast Scotland. Sci. Total Environ. 314-316, 289-302 (2003).

72. Moore, J. A. Surface transport of microorganisms by water. Biotechnology 15, 41-55 (1991).

73. Gibbs, S. G., Green, C. F., Tarwater, P. M. \& Scarpino, P. V. Airborne antibiotic resistant and nonresistant bacteria and fungi recovered from two swine herd confined animal feeding operations. J. Occup. Environ. Hyg. 1, 699-706 (2004).

74. Chapin, A., Rule, A., Gibson, K., Buckley, T. \& Schwab, K. Airborne multidrug-resistant bacteria isolated from a concentrated swine feeding operation. Environ. Health Perspect. 113, 137-142 (2005)

75. Elliott, P., Wakefield, J., Best, N. ¿ Briggs, D. Spatial epidemiology: Methods and Applications. (Oxford University Press, Oxford, 2000).

76. Lawson, A. B. Statistical Methods in Spatial Epidemiology. (John Wiley, Chichester 2001).

77. Hendrickx, G. et al. The spatial pattern of trypanosomosis prevalence predicted with the aid of satellite imagery. Parasitology 120, 121-134 (2000).

78. Kitron, U. \& Kazmierczak, J. J. Spatial analysis of the distribution of Lyme disease in Wisconsin. Am. J. Epidemiol. 145, 558-566 (1997).

79. Chadee, D. D. \& Kitron, U. Spatial and temporal patterns of imported malaria cases and local transmission in Trinidad. Am. J. Trop. Med. Hyg. 61, 513-517 (1999)

80. Kleinschmidt, I., Sharp, B., Mueller, I. \& Vounatsou, P. Rise in malaria incidence rates in South Africa: a small-area spatial analysis of variation in time trends. Am. J. Epidemiol. 155, 257-264 (2002)

81. Ali, M., Emch, M., Yunus, M. \& Sack, R. B. Are the environmental niches of Vibrio cholerae 0139 different from those of Vibrio cholerae O1 EI Tor? Int. J. Infect. Dis. 5, 214-219 (2001).

82. Ali, M., Emch, M., Donnay, J. P., Yunus, M. \& Sack, R. B. The spatial epidemiology of cholera in an endemic area of Bangladesh. Soc. Sci. Med. 55, 1015-1024 (2002).

83. Myaux, J., Ali, M., Felsenstein, A., Chakraborty, J. \& De Francisco, A. Spatial distribution of watery diarrhoea in children: identification of 'risk areas' in a rural community in Bangladesh. Health Place. 3 , 181-186 (1997)

84. Cifuentes, E., Mazari-Hiriart, M., Carneiro, F. Bianchi, F. \& Gonzalez, D. The risk of enteric diseases in young children and environmental indicators in sentinel areas of Mexico City. Int. J. Environ. Health Res. 12, 53-62 (2002).

85. Njemanze, P. C., Anozie, J., Ihenacho, J. O., Russell, M. J. \& Uwaeziozi, A. B. Application of risk analysis and geographic information system technologies to the prevention of diarrheal diseases in Nigeria. Am. J. Trop. Med. Hyg. 61, 356-360 (1999)
86. Tobler, W. R. A computer movie simulating urban growth in the Detroit region. Econ. Geograph. 46 234-240 (1970)

87. Singer, R. S., Case, J. T., Carpenter, T. E., Walker, R. L. \& Hirsh, D. C. Assessment of spatial and temporal clustering of ampicillin- and tetracyclineresistant strains of Pasteurella multocida and $P$. haemolytica isolated from cattle in California J. Am. Vet. Med. Assoc. 212, 1001-1005 (1998).

88. Metlay, J. P., Branas, C. C. \& Fishman, N. O. Hospital-reported pneumococcal susceptibility to penicillin. Emerg. Infect. Dis. 10, 54-59 (2004).

89. Ward, M. P. \& Carpenter, T. E. Techniques for analysis of disease clustering in space and in time in veterinary epidemiology. Prev. Vet. Med. 45, 257-284 (2000)

90. Singer, R. S., Reid-Smith, R. \& Sischo, W. M. Stakeholder position paper: Epidemiological perspectives on antibiotic use in animals. Prev. Vet. Med. 73, 153-161 (2006)

91. Diez-Roux, A. V. Bringing context back into epidemiology: variables and fallacies in multilevel analysis. Am. J. Public Health 88, 216-222 (1998).

92. Dohoo, I. R., Martin, S. W. \& Strynh, H. in Veterinary Epidemiologic Research. (Atlantic Veterinary College Inc., 2003).

93. Frost, L. S., Leplae, R., Summers, A. O. \& Toussaint, A. Mobile genetic elements: the agents of open source evolution. Nature Rev. Microbiol. 3 . 722-732 (2005).

94. Gogarten, J. P. \& Townsend, J. P. Horizontal gene transfer, genome innovation and evolution. Nature Rev. Microbiol. 3, 679-687 (2005).

95. Thomas, C. M. \& Nielsen, K. M. Mechanisms of, and barriers to, horizontal gene transfer between bacteria. Nature Rev. Microbiol. 3, 711-721 (2005).

96. Handelsman, J. Metagenomics: application of genomics to uncultured microorganisms. Microbiol. Mol. Biol. Rev. 68, 669-685 (2004)

97. Riesenfeld, C. S., Goodman, R. M. \& Handelsman, J. Uncultured soil bacteria are a reservoir of new antibiotic resistance genes. Environ. Microbiol. 6, 981-989 (2004).

98. Franklin, R. B. \& Mills, A. L. Multi-scale variation in spatial heterogeneity for microbial community structure in an eastern Virginia agricultural field. FEMS Microbiol. Ecol. 44, 335-346 (2003).

99 Yu, Z., Michel, F. C. Jr, Hansen, G., Wittum, T. \& Morrison, M. Development and application of realtime PCR assays for quantification of genes encoding tetracycline resistance. Appl. Environ. Microbiol. 71 6926-6933 (2005)

100. Smith, M. S. et al. Quantification of tetracycline resistance genes in feedlot lagoons by real-time PCR Appl. Environ. Microbiol. 70, 7372-7377 (2004).

101. Hayes, J. R., Wagner, D. D., English, L. L., Carr, L. E. \& Joseph, S. W. Distribution of streptogramin resistance determinants among Enterococcus faecium from a poultry production environment of the USA J Antimicrob. Chemother. 55, 123-126 (2005).

\section{Acknowledgements}

We thank Tim Boyer, Janet Anderson and the reviewers for many helpful suggestions in improving the manuscript. We also thank Jeffrey Bender for supplying the Minnesota STEC dataset that was used in this manuscript. R.S.S. was partially supported by a United States Department of Agriculture National Research Initiative Competitive Grant.

Competing interests statement

The authors declare no competing financial interests.

\section{DATABASES}

The following terms in this article are linked online to:

Entrez Genome Project: http://www.ncbi.nlm.nih.gov/ entrez/query.fcgi?db=genomeprj

Enterococcus faecium|Escherichia coli

FURTHER INFORMATION Randall S. Singer's homepage: http://www.singerlab.umn.edu/ Michael P. Ward's homepage:

http://www.cvm.tamu.edu/vibs/vetepi/people.htm

Access to this links box is available online. 


\section{CORRIGENDUM}

\section{Can landscape ecology untangle the complexity of antibiotic}

resistance?

Randall S. Singer, Michael P. Ward and George Maldonado

Nature Reviews Microbiology 4, 943-952 (2006)

In the above article there was an error in the equation shown in Box 1. This error had no impact on the graph shown in the Box, but the authors wish to apologise to readers for any confusion caused. The correct equation is shown below; additionally, in the box table, the value for parameter $b$ (the selection pressure exerted by antibiotic 2 , which is expressed as the proportion of the selection pressure exerted by antibiotic 1 ) should have read $0-60 \%$.

$P($ resistance $\mid$ use $)=\mu \times a$

$P($ resistance $\mid$ no use $)=\mu(1+b[a-1])$

$\mathrm{RR}=\frac{P(\text { resistance } \mid \text { use })}{P(\text { resistance } \mid \text { no use })}$ 